Int J Microcirc 1995;15:333-334

\title{
Subject Index, Vol. 15, 1995
}

Vol. 15, Supplement 1, has its own Subject Index

Acetylcholine 283 Adhesion 255

- $\quad$ molecules 255,301

Adrenalectomy 309

Aggregation 255

Aging 231

Aldosterone 309

Amiloride 265

Anesthesia 163

Angiogenesis Ill, 181

Ankle skin 65

Aorta occlusion 271 Arteriolar internal diameter 170 Arteriole(s) 28, 48, 309 Arteriovenous shunt flow 92 ATP 1

Blackfoot disease 21 Blood circulation 325

flow 250

-, microvascular 170

loss 316

rheology 250 Bradykinin 293 Brain microvessels 1 Breast blood flow 125

- $\quad$ cancer, detection and prognosis

125

Ca2+channel blockers 92 Capillaries 250 -, contractility 238

density 65,Ill,218,271

deviations 287

diameter 238, 271

flow 92

hemodynamics/RBC flow 223

network structure/function 223

obstruction 255

perfusion 244 Capillaroscopy 65 Capillary microscopy, vital 287

- morphology 287

Capsaicin 1

Cardiac transplant recipients 117 Catalase 155 Cerebral blood flow 37 Cheek pouch, hamster 293 Chorioallantoic membrane 181 Chronic arseniasis 21

- $\quad$ stimulation Ill

Cold-induced vasodilatation 92

Computer-assisted analysis 244 Critical limb ischemia 60

Dermal microcirculation 131 Dexamethasone 309 Diabetic hamsters 293

microangiopathy 193

retinopathy 193 Disuse 231 
Dorsal skinfold chamber 85

Edema/oedema 137,231 Electron microscopy 43, 155 Embryo, chick 186 Endothelial cells 1,43 cell-stimulating angiogenic factor Ill Endothelin(s) 48

receptor antagonists 48 Endothelin 1 Endothelin-1 48 Endothelin-3 48 Endothelium 75, 80, 238, 255 Endotoxic shock 170 Erythrocytes 255

Flow cytometry 85

Flowmotion 28

Fluorescence microscopy, intravital 85

- $\quad$ videomicroscopy 193

Functional capillary density 238, 250

Glycosaminoglycans 181 Gracilis muscle 155

Haemorrhagic shock 43

Hamster 244

Heart failure 137

Hemoglobin oxygenation 131

Hemorrhage 163

Heparin 181

Heterogeneity 244

Histamine 293

Hypercapnia 316

Hyperemia, reactive 75

Hyperemic response 231

Hypertonic saline-dextran solution 265

Hypocapnia 277

Iloprost 60

Inflammatory mediators 301 Internal maxillary artery 92 Intestinal obstruction 325

Intracranial pressure, high 316 Intravital microscopy 271 Isch(a)emia 155,231,255,325

reperfusion 293

- injury 14

Knee surgery 14

Laser Doppler 65

- flowmetry 21,28,37,316

- flux(i)metry 10,137,277

- imager 125

- perfusion measurements 117

- scanning 37 Leukocyte 265

adhesion 293, 301

staining 85 Leukotriene B4 293

Macromolecular permeability 293 Mesenteric venules, rat 301 Microaneurysms 193

Microcirculation 37, 137, 163,244,250,

265 -, cerebral 316 Microvascular reactivity 117

- $\quad$ skin blood flow 10

Model analysis 265

Monoclonal antibodies 301

Muscle, skeletal 163 
Myocardial infarction, human 80

Myocardium 43

Nailfold 287 Nasal mucosa 92 Nifedipine 92 Nitric oxide 170,283 Nitroprusside 283 No-reflow phenomenon 265

Oxygen 271

concentration 186

radicals 155

Papaverine 309 PD 14289348 PD 14795348 Perfusion 231

Peripheral arterial disease 60 Platelets 255

Plethysmography, mercury in silastic strain gauge 14

Postocclusive reactive hyperemia 10 Pressure 238

Primary biliary cirrhosis 75 Prostacyclin 170

Rabbits 28

Reactive hyperaemia 53

Recombinant human erythropoietin 283

Red blood cell(s) 250

- -, flow redistribution 223

- -, velocity 231 Reflection oxymetry 131 Regulation 218 Reperfusion 155 Rhodamine 6G 85

S5682 293 Shock 255 Shunts 218

Simulation study 37 Single-fibre probe 53 Skeletalmuscle(s) 28, HI

- microcirculation 223 Small intestine 325 Sodium fluorescein 10 Soluble E-selectin 80 Spatial heterogeneity 223

variability 53 Spectroscopy 131 Substance P 1 Superoxide dismutase 155

Temporal heterogeneity 223 Thermal stress 21 Thermography 283 Tissue perfusion imaging 125

- $\quad$ trauma 53

Tissue-type plasminogen activator 75

Tourniquet 14 Transcutaneous CO2 60 - p02 60 Tumour blood flow 125

Vascularization 186 Vasoconstriction 48 Vasodilatation 277 Vasomotion 14,28, 170 Vasopressin 1

Venoarteriolar response 137 Venous ulcer site 65 Verapamil 92 Videomicroscopy 10

Volumotion 14 Von Willebrand factor 75

Yolk sac membrane 186

334

Subject Index Vol. 15, 1995 